\title{
Durvalumab-induced diabetic ketoacidosis followed by hypothyroidism
}

\author{
Shivani Patel 1,3 , Venessa Chin ${ }^{2,4}$ and Jerry R Greenfield $1,3,4$ \\ 1Department of Diabetes and Endocrinology, St Vincent's Hospital Sydney, Darlinghurst, New South Wales, Australia, \\ ${ }^{2}$ The Kinghorn Cancer Centre, Sydney, New South Wales, Australia, ${ }^{3}$ Diabetes and Metabolism, Garvan Institute of \\ Medical Research, Sydney, New South Wales, Australia, and 4St Vincent's Clinical School, UNSW Sydney, Darlinghurst, \\ New South Wales, Australia
}

Correspondence should be addressed to S Patel

Email

shivani.patel146@gmail.com

\section{Summary}

Durvalumab is a programmed cell death ligand 1 inhibitor, which is now approved in Australia for use in non-smallcell lung and urothelial cancers. Autoimmune diabetes is a rare immune-related adverse effect associated with the use of immune checkpoint inhibitor therapy. It is now being increasingly described reflecting the wider use of immune checkpoint inhibitor therapy. We report the case of a 49-year-old female who presented with polyuria, polydipsia and weight loss, 3 months following the commencement of durvalumab. On admission, she was in severe diabetic ketoacidosis with venous glucose: $20.1 \mathrm{mmol} / \mathrm{L}, \mathrm{pH}: 7.14$, bicarbonate $11.2 \mathrm{mmol} / \mathrm{L}$ and serum beta hydroxybutyrate: $>8.0 \mathrm{mmol} / \mathrm{L}$. She had no personal or family history of diabetes or autoimmune disease. Her HbA1c was $7.8 \%$ and her glutamic acid decarboxylase (GAD) antibodies were mildly elevated at $2.2 \mathrm{mU} / \mathrm{L}$ (reference range: $<2 \mathrm{mU} / \mathrm{L}$ ) with negative zinc transporter 8 (ZnT8) and islet cell (ICA) antibodies. Her fasting C-peptide was low at 86 pmol/L (reference range: 200-1200) with a corresponding serum glucose of $21.9 \mathrm{mmol} / \mathrm{L}$. She was promptly stabilised with an insulin infusion in intensive care and discharged on basal bolus insulin. Durvalumab was recommenced once her glycaemic control had stabilised. Thyroid function tests at the time of admission were within normal limits with negative thyroid autoantibodies. Four weeks post discharge, repeat thyroid function tests revealed hypothyroidism, with an elevated thyroid-stimulating hormone (TSH) at $6.39 \mathrm{mIU} / \mathrm{L}$ (reference range: 0.40-4.80) and low free T4: $5.9 \mathrm{pmol} / \mathrm{L}$ (reference range: 8.0-16.0). These findings persisted with repeat testing despite an absence of clinical symptoms. Treatment with levothyroxine was commenced after excluding adrenal insufficiency (early morning cortisol: $339 \mathrm{nmol} / \mathrm{L}$ ) and hypophysitis (normal pituitary on MRI).

\section{Learning points:}

- Durvalumab use is rarely associated with fulminant autoimmune diabetes, presenting with severe DKA.

- Multiple endocrinopathies can co-exist with the use of a single immune checkpoint inhibitors; thus, patients should be regularly monitored.

- Regular blood glucose levels should be performed on routine pathology on all patients on immune checkpoint inhibitor.

- Clinician awareness of immunotherapy-related diabetes needs to increase in an attempt to detect hyperglycaemia early and prevent DKA.

\section{Background}

Immune checkpoint inhibitors (ICIs) consist of monoclonal antibodies which bind to various immune checkpoint proteins, including cytotoxic T-lymphocyte antigen 4 (CTLA-4), programmed cell death protein 1 (PD-1) and its ligand (PD-L1). In oncology, their desired target effect is to upregulate an immune response towards 
cancer cells. However, inhibition of immune checkpoints may result in unwanted immunotherapy-related adverse effects (irAE) as normal cells are affected.

Immune-related adverse effects are being increasingly recognised as the use of ICIs rapidly rises (1). More agents are becoming available, as clinical indications for their use in advanced cancers continue to increase. Endocrinopathies are common, namely, thyroid dysfunction and hypophysitis. Primary adrenal insufficiency has been rarely described (2). They differ from other irAE in that they are irreversible and often require long-term management. Thyroid dysfunction has been reported in up to $14 \%$ of patients following commencement of immunotherapy (1). This risk is heightened with the use of multiple immunotherapy agents (3). Autoimmune diabetes is rare. Its pathophysiology remains to be completely understood (2).

To date, most endocrinopathies described have been associated with the use of nivolumab, pembrolizumab and ipilimumab. Durvalumab is a newer human MAB that binds to PD-L1. The PACIFIC trial showed that its use significantly prolonged progression-free survival and overall survival compared to placebo alone in patients with stage III, non-small-cell lung cancer (NSCLC) after definitive chemoradiation (4). In this study, new-onset diabetes occurred in one patient $(<0.1 \%)$. In Australia, it is currently approved for use in both stage III NSCLC and urothelial cancers. We report a case of fulminant diabetic ketoacidosis associated with durvalumab use in nonsmall-cell lung cancer followed by subsequent primary hypothyroidism.

\section{Case presentation}

A 49-year-old female presented to her routine oncology appointment with a 1-week history of lethargy, polyuria, polydipsia and blurred vision. She denied abdominal pain, nausea or vomiting. This was on a background of locally advanced lung adenocarcinoma, which was being treated with adjuvant durvalumab, commenced 3 months prior. She had shown good radiological response to her concurrent chemoradiation and immunotherapy treatment.

She had no prior personal or family history of diabetes or autoimmune disease. She had not been on recent glucocorticoid therapy. During her appointment, her random finger prick glucose was elevated at $21.9 \mathrm{mmol} / \mathrm{L}$, with ketones $6.7 \mathrm{mmol} / \mathrm{L}$.

She was subsequently admitted to hospital and an initial blood gas confirmed diabetic ketoacidosis (DKA) with serum glucose: $20.1 \mathrm{mmol} / \mathrm{L}, \mathrm{pH}: 7.14$, bicarbonate: $11.2 \mathrm{mmol} / \mathrm{L}$ and serum beta hydroxybutyrate: $>8.0$ $\mathrm{mmol} / \mathrm{L}$. She was otherwise haemodynamically stable with a normal clinical examination.

\section{Investigation}

Initial $\mathrm{HbA} 1 \mathrm{c}$ on admission was elevated at $7.8 \%$. She was biochemically euthyroid, with serum TSH 0.95 $\mathrm{mIU} / \mathrm{L}$. Serum fasting C-peptide was low at $86 \mathrm{pmol} / \mathrm{L}$ (reference range (RR): 200-1200) with a corresponding serum glucose of $21.9 \mathrm{mmol} / \mathrm{L}$. GAD antibody was weakly positive at $2.2 \mathrm{U} / \mathrm{mL}(\mathrm{RR}: \geq 2)$; ICA and ZnT8 antibodies were negative. Prior random glucose measurements were not documented.

\section{Treatment}

Her DKA resolved with $21 \mathrm{~h}$ of an insulin infusion. She was subsequently transitioned onto s.c. insulin and discharged on basal bolus insulin.

\section{Outcome and follow-up}

Blood glucose levels were well controlled on basal bolus insulin. Her hyperglycaemia-associated symptoms had significantly improved. She required 20 units of glargine insulin (Lantus) and 1 unit of insulin aspart (NovoRapid) for every $10 \mathrm{~g}$ of carbohydrates. Durvalumab was withheld until her glycaemic control stabilised. Repeat C-peptide 6 weeks later was undetectable at $<33 \mathrm{pmol} / \mathrm{L}$ with a serum glucose of $16.5 \mathrm{mmol} / \mathrm{L}$, confirming insulin deficiency.

Four weeks following hospital admission, repeat thyroid function revealed hypothyroidism with serum TSH: $6.39 \mathrm{mIU} / \mathrm{L}$, free T4: $5.9 \mathrm{pmol} / \mathrm{L}$ with negative thyroid TPO and thyroglobulin antibodies. Cortisol at $10.30 \mathrm{am}$ was low at $166 \mathrm{nmol} / \mathrm{L}$ with ACTH $2.1 \mathrm{pmol} / \mathrm{L}$ (Table 1 ). A short Synacthen test was not performed at the time (due to a nationwide shortage of Synacthen and an adequate repeat early morning cortisol at $339 \mathrm{nmol} / \mathrm{L}$ ). An MRI pituitary excluded hypophysitis. She was subsequently commenced on levothyroxine $50 \mu \mathrm{g}$ daily.

\section{Discussion}

Thyroid dysfunction has been well described to be the most common irAE across all classes of ICI therapy (1). Autoimmune diabetes on the other hand, is rarely associated with PD-1 and PD-L1 blockade $(2,5)$. 
Table 1 Thyroid function and cortisol levels post discharge.

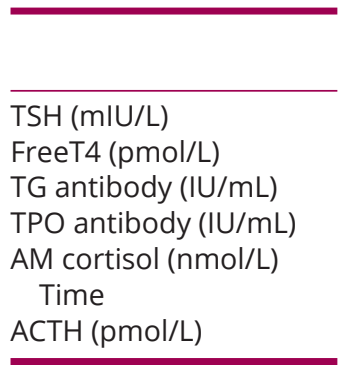

\begin{tabular}{cc}
\hline Day 56 \\
0.41
\end{tabular}

Day post durvalumab commencement

\begin{tabular}{|c|c|c|c|}
\hline Day 119 & Day 127 & Day 133 & Day 161 \\
\hline 6.39 & 8.69 & 11.8 & 6.29 \\
\hline 5.9 & 7.9 & 7.1 & 9.5 \\
\hline 166 & 234 & 339 & 267 \\
\hline $\begin{array}{c}10: 32 \mathrm{~h} \\
2.1\end{array}$ & $10: 57 \mathrm{~h}$ & 08:10 h & $08: 55 \mathrm{~h}$ \\
\hline
\end{tabular}

ACTH, adrenocorticotropic hormone; TG, thyroglobulin; TPO, thyroid peroxidase; TSH, thyroid-stimulating hormone.

Endocrinopathies secondary to newer agents such as durvalumab use have been not been as well described.

Our patient presented with severe DKA within 3 months of initiation of durvalumab with fulminant onset as reflected by clinical symptoms, low C-peptide and $\mathrm{HbA} 1 \mathrm{c}$ of $7.8 \%$ with weakly positive autoantibodies. This is in keeping with previously reported literature. A recent systematic review and meta-analysis of PD-1and PD-L1-associated type 1 diabetes reviewed 71 cases (3). Seventy six percent of these cases presented with diabetic ketoacidosis. The median time to onset was 49 days with $71 \%$ presenting within 3 months of initiation of immunotherapy. As in our case, mean HbA1c was $7.8 \%$ with no correlation between HbA1c and time to onset. This is reflective of the rapid onset and relatively short period of hyperglycaemia prior to presentation. Prior glucose measurements were not performed despite guidelines and local protocols recommending routine monitoring in these patients (6).

Type 1 diabetes-associated antibodies have been reported to be positive in up to $50 \%$ of cases $(3,5)$. Whilst this patient's GAD antibodies were only mildly elevated, it has been noted that patients with positive GAD antibodies have been found to have a shorter duration to diabetes onset and higher incidence of diabetic ketoacidosis compared to patients without antibodies at presentation $(3,5)$. GAD antibodies may become positive over time. Merchand et al. analysed six cases of immunotherapy-induced diabetes in which one patient had borderline IA2 antibody elevation. This patient had a transient form of diabetes in comparison to those with fulminant diabetes who were all GAD, IA2 and ZnT8 antibody negative (7).

The pathophysiology of immunotherapy-related diabetes remains unclear. It has been proposed that the aetiology differs from classic antibody-positive type 1 diabetes with rapid beta cell destruction due to cell-mediated toxicity (3). Other contributing risk factors and biomarkers predisposing certain individuals to diabetes are yet to be identified.

The development of other endocrinopathies either prior to or concurrent to the development of diabetes has been described in up to $44 \%$ of patients (5). This risk is heightened with combination therapy (3). The majority of these patients had primary thyroid dysfunction (hypothyroidism or thyroiditis) (5). Nevertheless, as seen in our patient, multiple endocrinopathies may occur following use of a single ICI. These may co-exist or present in succession, highlighting the need for ongoing monitoring.

The association between development of an irAE and oncological response remains controversial. Horvat et al. found that overall survival and time to treatment failure in melanoma patients treated with ipilimumab were not affected by the presence of irAE (8). Meanwhile Downey et al. found that majority of patients who achieved partial or complete response developed some form of irAE, with more severe irAEs in all patients who achieved complete response (9). Overall, whilst the presence of an irAE suggests immune activation, they do not necessarily indicate effective immune blockade. Certain adverse effects, such as vitiligo, may be more strongly associated with treatment efficacy (10).

In summary, this case adds to the emerging literature that immunotherapy may precipitate autoimmune diabetes, presenting with severe, life-threatening DKA. All patients on ICI therapy should be screened regularly with serum glucose levels in an attempt to detect hyperglycaemia prior to the development of DKA. Whilst clinician awareness of this adverse effect needs to increase, forewarning patients remains challenging without increasing angst.

In patients with an established immune-related endocrinopathy, regular monitoring for other possible 
endocrinopathies should continue, in line with local protocols and guidelines. Future research is needed to identify risk factors and biomarkers for the development of immunotherapy-related diabetes and to investigate the relationship between immune-related endocrinopathy and response to cancer therapy.

\section{Patient's perspective}

Before I started my course of durvalumab treatment for Stage III nonsmall-cell lung cancer, I was informed of the many side effects, the most common (amongst others) affecting thyroid function. Diabetes was not one of them.

When I was about 3 months into my treatment, I noticed that my eyesight had changed. Long distance vision became blurry but because glasses corrected this, I was told that there was no cause for concern. Soon after, I experienced unbelievable fatigue which I put down to the treatment but when this was coupled with unquenchable thirst, frequent urination and weight loss I had a feeling something was not right. I knew the symptoms of diabetes and these were textbook symptoms. Upon an urgent request from my oncologist to see her, a prick test confirmed high glucose levels. Fortunately, I was immediately referred to the Diabetes Centre at St Vincent's Hospital. After seeing Drs Patel and Greenfield and, with further testing, I was fast tracked into emergency and ICU with DKA where I was given IV insulin and other necessary fluids to return my levels to normal.

I knew so little about diabetes and definitely had no idea that it could be a side effect of the immunotherapy. I certainly did not realise the lifethreatening implications of diabetes and DKA and am very fortunate I was seen to as quickly as I was. I did not have any nausea or vomiting, typical of DKA, a fact which potentially could have delayed the urgency with which I was attended to.

As it is such a rare side effect, there is not much known about it but for now it is being treated as Type 1, insulin dependent and probably permanent. With education and a steep learning curve I have come to know a new normal. It is so much more than I ever imagined; it is a $24 / 7$ job, constantly measuring glucose levels and calculating how much insulin is needed for the amount of carbs about to be consumed. Then, a few weeks after the diabetes diagnosis, my blood results indicated that my thyroid function was out of the normal range, indicating hypothyroidism. I had experienced no symptoms of fatigue or headaches which I informed Dr Patel of when asked. In any event, I was prescribed levothyroxine which is to be taken each morning and have been told will probably be lifelong. To date, this diagnosis has not had any effect on me.

I have come to learn that there are so many factors (not just sugar vs insulin) which could affect glucose levels, from stress to the weather to coming down with a cold, and with the addition of an unknown element of the immunotherapy, the unpredictability of it can be quite exhausting. Honestly, at times it feels more difficult to manage than the cancer itself because at least the treatment plan for that is pre-determined. The reality though, is now that it's been diagnosed, it is a manageable and controllable disease and the main outcome is that I can continue with and complete my course of immunotherapy which is fighting a much more severe and serious illness.

\section{Declaration of interest}

The authors declare that there is no conflict of interest that could be perceived as prejudicing the impartiality of this case report.

\section{Funding}

This research did not receive any specific grant from any funding agency in the public, commercial or not-for-profit sector.

\section{Patient consent}

Written informed consent was obtained from the patient for publication of the submitted article.

\section{Author contribution statement}

S P , V C and J R G treated the patient and reviewed the data. S P wrote the manuscript. V C and J R G reviewed the manuscript.

\section{References}

1 Delivanis DA, Gustafson MP, Bornschlegl S, Merten MM, Kottschade L, Withers S, Dietz AB \& Ryder M. Pembrolizumabinduced thyroiditis: comprehensive clinical review and insights Into underlying involved mechanisms. Journal of Clinical Endocrinology and Metabolism 2017102 2770-2780. (https://doi.org/10.1210/ jc.2017-00448)

2 Osamah AH, Julia I, Shahzad A, Tommy Kyaw T, Seamus S $\&$ John HM. A case of pembrolizumab-induced severe DKA and hypothyroidism in a patient with metastatic melanoma. Endocrinology, Diabetes and Metabolism Case Reports 20192019 $18-153$.

3 Akturk HK, Kahramangil D, Sarwal A, Hoffecker L, Murad MH \& Michels AW. Immune checkpoint inhibitor-induced Type 1 diabetes: a systematic review and meta-analysis. Diabetic Medicine 201936 1075-1081. (https://doi.org/10.1111/dme.14050)

4 Antonia SJ, Villegas A, Daniel D, Vicente D, Murakami S, Hui R, Yokoi T, Chiappori A, Lee KH, de Wit M, et al. Durvalumab after chemoradiotherapy in stage III non-small-cell lung cancer. New England Journal of Medicine 2017377 1919-1929. (https://doi. org/10.1056/NEJMoa1709937)

5 Stamatouli AM, Quandt Z, Perdigoto AL, Clark PL, Kluger H, Weiss SA, Gettinger S, Sznol M, Young A, Rushakoff R, et al. Collateral damage: insulin-dependent diabetes induced with checkpoint inhibitors. Diabetes 201867 1471-1480. (https://doi. org/10.2337/dbi18-0002)

6 Haanen JBAG, Carbonnel F, Robert C, Kerr KM, Peters S, Larkin J, Jordan K \& ESMO Guidelines Committee. Management of toxicities from immunotherapy: ESMO Clinical Practice Guidelines for diagnosis, treatment and follow-up. Annals of Oncology 201829 (Supplement_4) iv264-iv266. (https://doi.org/10.1093/annonc/mdy162)

7 Marchand L, Thivolet A, Dalle S, Chikh K, Reffet S, Vouillarmet J, Fabien N, Cugnet-Anceau C \& Thivolet C. Diabetes mellitus induced by PD-1 and PD-L1 inhibitors: description of pancreatic endocrine and exocrine phenotype. Acta Diabetologica 201956 441-448. (https://doi.org/10.1007/s00592-018-1234-8)

8 Horvat TZ, Adel NG, Dang TO, Momtaz P, Postow MA, Callahan MK, Carvajal RD, Dickson MA, D'Angelo SP, Woo KM, et al. Immunerelated adverse events, need for systemic immunosuppression, and effects on survival and time to treatment failure in patients with melanoma treated with ipilimumab at Memorial Sloan Kettering Cancer Center. Journal of Clinical Oncology 201533 3193-3198. (https://doi.org/10.1200/JCO.2015.60.8448)

9 Downey SG, Klapper JA, Smith FO, Yang JC, Sherry RM, Royal RE, Kammula US, Hughes MS, Allen TE, Levy CL, et al. Prognostic factors 
related to clinical response in patients with metastatic melanoma treated by CTL-associated antigen- 4 blockade. Clinical Cancer Research 200713 6681-6688. (https://doi.org/10.1158/1078-0432. CCR-07-0187)
10 Postow MA, Sidlow R \& Hellmann MD. Immune-related adverse events associated with immune checkpoint blockade. New England Journal of Medicine 2018378 158-168. (https://doi.org/10.1056/ NEJMra1703481)

Received in final form 10 October 2019

Accepted 21 November 2019 